\title{
Formación profesional en el campo de la Educación en el contexto actual: Aristas y puntos ciegos
}

Professional formation in the field of Education in the current context: edges and blind spots

\author{
Volumen 19, Número 2 \\ Mayo-Agosto \\ pp. $1-25$
}

Flora Eugenia Salas Madriz

Cite este documento así

Salas Madriz, Flora Eugenia. (2019). Formación profesional en el campo de la Educación en el contexto actual: Aristas y puntos ciegos. Revista Actualidades Investigativas en Educación, 19(2), 1-25. Doi. 10.15517/aie.v19i2.37024 


\title{
Formación profesional en el campo de la Educación en el contexto actual: Aristas y puntos ciegos \\ Professional formation in the field of Education in the current context: edges and blind spots
}

\section{Flora Eugenia Salas Madriz}

\begin{abstract}
Resumen: En este ensayo se examina la formación profesional en Educación en un contexto global y local complejo, marcado por el cambio social, económico, político y cultural, donde fuerzas tradicionales y transformadoras están en tensión; entre ellas, la visión empírico-analítica de la ciencia moderna y los nuevos enfoques teórico-epistemológicos posmodernos. Al respecto, se propone que la formación profesional en Educación se debe analizar a partir de paradigmas más ajustados a las características de los fenómenos sociales contemporáneos, cuya característica más significativa es la complejidad creciente. A partir de los aportes teóricoepistemológicos y metodológicos de la Teoría de los Sistemas Sociales de Niklas Luhmann y la Teoría de la Complejidad de Edgar Morin, se construye un modelo para la descripción, comprensión y explicación de la profesionalización docente, la cual se entiende como un sistema que contiene en su estructura y funciones, los subsistemas de la formación de grado, posgrado y a lo largo de la vida laboral, que expresa, a su vez, relaciones internas, entre subsistemas y con la sociedad (entorno). Se deriva del modelo que de la forma, relaciones y desarrollo de los niveles de formación docente es posible planificar, controlar, administrar y evaluar el desempeño profesional docente $y$, en consecuencia, el sistema de formación como un todo; razón por la cual, el modelo propuesto sirve de referente para identificar áreas de mejora, así como problemas estructurales y funcionales de la profesionalización docente, que no es posible detectar ni explicar, a partir de un enfoque empírico-analítico del fenómeno.
\end{abstract}

Palabras clave: formación profesional, educación, teoría de la educación, teoría de la complejidad.

\begin{abstract}
This essay examines professional formation in education in education in a complex global and local context, marked by social, economic, political and cultural change, where traditional and transforming forces are in tension; among them, the empirical-analytical vision of modern science and the new postmodern theoreticalepistemological approaches. In this regard, it is proposed that professional training in education should be analyzed from paradigms more adjusted to the characteristics of social phenomena in our time, whose most significant feature is the increasing complexity. From the theoretical-epistemological and methodological contributions of the Theory of Social Systems by Niklas Luhmann and the Theory of Complexity by Edgar Morin, a model for the description, understanding and explanation of teacher professionalization is constructed, where it is understood as a system that contains in its structure and functions, the subsystems of the undergraduate, graduate and throughout the working life, which expresses, in turn, internal relations, between subsystems and with society (environment). It is derived from the model that of the form, relationships and development of teacher training levels it is possible to plan, control, manage and evaluate the professional performance of teachers and, consequently, the training system as a whole; For this reason, the proposed model serves as a reference to identify areas for improvement, as well as structural and functional problems of teacher professionalization, which cannot be detected or explained, based on an empirical-analytical approach to the phenomenon.
\end{abstract}

Key words: professional formation, education, educational theory, complexity theory.

\footnotetext{
1 Docente e Investigadora de la Universidad de Costa Rica, en la Vicerrectoría de Docencia, Costa Rica. Doctora en Educación por la Universidad de Costa Rica.
}

Dirección electrónica: flora.salas@ucr.ac.cr

Ensayo recibido: $1^{\circ}$ de octubre, 2018

Enviado a corrección: 25 de febrero, 2019

Aprobado: $1^{\circ}$ de abril, 2019 


\section{Introducción: el contexto}

La formación profesional en Educación es un componente clave del fenómeno educativo, el cual incide en las características y atributos del proceso de enseñanza y aprendizaje, así como en la experiencia educativa del estudiantado. Por ello, es un tema recurrente en diversas conferencias mundiales organizadas por la United Nations Educational, Scientific and Cultural Organization (Unesco) en los últimos 30 años del siglo XX (García, 1999), en un debate que persiste hasta nuestros días, debido a los cambios en el contexto mundial de la década de 1990, cuando la formación profesional docente cobró la dimensión de "tarea prioritaria"; ello, en respuesta a la presión social, política y económica por transformaciones capaces de llevar la educación al nivel de las demandas sociales emergentes, impulsadas por la Globalización (Beck, 1998), la Sociedad de la Información y el Conocimiento (SIC) (Castells, 2000a, 2000b, 2001), y la Posmodernidad (Beck, Giddens y Lash, 2001; Luhmannn, 1997).

En el contexto descrito, eventos como la mundialización de la economía de mercado de corte postindustrial (Bell, 1994a, 1994b), el desarrollo y mejora de las tecnologías de la información y la comunicación (TIC), que impactan a toda la sociedad (Castells, 2000a, 2000b, 2001), y el avance de la cultura posmoderna, donde el individuo y el individualismo adquirieron una dimensión sin precedentes en la historia de la humanidad (Gidens, 1995), son componentes esenciales de la crisis de las instituciones claves de la Era Moderna: el Estado social de Derecho, la familia tradicional, la religiosidad, la empresa privada, el comercio local y mundial, y, con particular fuerza y apremio, los sistemas educativos públicos y privados.

En razón de la naturaleza y magnitudes del cambio que experimentamos en la actualidad -que, como se mencionó, es mundial y se impone a todas las naciones, independientemente de su nivel de desarrollo socioeconómico- vivimos en un "tiempo extraño", resultante de una fase de transición de un período histórico que pierde fuerza y se disloca, pero que también se radicaliza en algunas de sus manifestaciones ideológicas y prácticas, hacia otro que prospera y se articula, ganando resonancia y legitimidad social. Debido a que esta es una de las principales características de nuestro tiempo, las formas de organización socioeconómica, política y cultural, propias de la Sociedad Industrial conviven, no sin tensiones, con formas incipientes e inéditas que son distintivas de la Sociedad Postindustrial y posmoderna (Bell, 1994a, 1994b). Inevitablemente, esos modos de 
organización social tan distintos entre sí, van en curso de colisión e inducen crisis profundas; peor aún, provocan "crisis en el manejo de las crisis" e ingobernabilidad (Offe, 1990) por el nivel de incertidumbre que provoca que muchas de las soluciones ejemplares del pasado ya no funcionen, mientras la realidad nos abruma con problemas sociales viejos y nuevos, para los cuales no parece haber una respuesta satisfactoria.

Para estudiar y comprender la formación profesional docente en el contexto local y mundial imperante, en este ensayo se argumenta sobre la necesidad de recurrir a marcos teórico-metodológicos que permitan el estudio de los fenómenos complejos, y se sostiene la tesis de que la formación docente se puede entender como un sistema estructurado en los subsistemas de grado, posgrado y a lo largo de la vida laboral que, funcionalmente, mantiene relaciones internas, entre subsistemas y con la sociedad (entorno). En consecuencia, se afirma que de la forma en la que se desarrollen los subsistemas de la formación profesional docente depende el desempeño del profesorado y el del sistema de formación como un todo.

Por lo anterior, se propone un modelo de la formación profesional docente, a partir del enfoque sistémico-complejo, entendido como un marco de referencia teórico-epistemológico alternativo al empírico-analítico, pertinente para la comprensión de fenómenos de alta complejidad. En el modelo, también se hace referencia a los componentes críticos del sistema de formación profesional en educación, a fin de plantear algunas tesis sobre los enlaces entre los distintos niveles de formación, así como mecanismos de articulación entre ellos y la sociedad que, en tanto parte de un modelo general, resultan útiles para estudiar cualquier sistema de formación profesional en el campo de la educación en sociedades complejas.

\section{La epistemología de la complejidad y la teoría de los sistemas sociales: Un nuevo marco teórico de referencia para atender los problemas en las sociedades posmodernas}

La epistemología clásica desarrollada en la filosofía griega describe y explica la relación de conocimiento -que es una abstracción relativamente simple-, mediante la cual se representa la producción del conocimiento científico. En sus orígenes, se expresa en la relación sujeto cognoscente/objeto de estudio, donde se asumen supuestos teóricos -marcos explicativos y conceptuales previos sobre el objeto de estudio-, métodos y técnicas 
de investigación, y resultados de investigación coherentes con las teorías y metodologías de referencia y, en consecuencia, la solución al problema en estudio. En ella, también, se asume que la relación "sujeto/objeto" está descontextualizada, es objetiva, y se valida y cobra sentido en sí misma, como si fuera un sistema cerrado; es decir, un sistema para el cual "el entorno no tiene ningún significado o que sólo tiene significado a través de canales específicos" (Luhmann, 1998, p. 31).

En la epistemología de la complejidad -que incluye el enfoque de sistemas y las ciencias de la complejidad-, se entiende que el conocimiento científico conforma un sistema abierto y que es un proceso contextualizado y determinado sociohistóricamente, donde el sujeto cognoscente y el objeto de estudio no son entidades aisladas que se legitiman y agotan en sí mismas, sino sistemas que conforman, a su vez, un sistema de conocimiento², donde el objeto no es una parte aislada del todo, sino que está constituido por una red de elementos internos y relaciones consigo mismo y el entorno; esto significa que "Todo objeto posible existe solo porque algún sistema lo constituye en cuanto unidad" (Corsi, Esposito y Baraldi, 2006, p. 160). Es decir, tiene interrelaciones y organización interna, y se vincula con el contexto en un proceso de mutua determinación.

Por su parte, el sujeto cognoscente no es "objetivo" ni está descontextualizado, sino que es resultado de su realidad sociohistórica y su biografía, así como de su biología, psique y procesos cognitivos y, por ende, solamente puede actuar como observador ${ }^{3} /$ conceptuador en el marco de referencia del avance del conocimiento científico de que se trate.

De acuerdo con la epistemología sistémico-compleja, no existe un sujeto cognoscente ni un objeto de estudio en el sentido de la epistemología clásica, sino un "circuito explicativo todo/partes [...]. Así, el sistema [objeto del conocimiento científico] debe ser concebido según

\footnotetext{
2 "La ciencia es un sistema funcionalmente diferenciado al interior de la sociedad, que opera con base en el medio de comunicación verdad. En relación con la sociedad global, la ciencia desarrolla la función de construir y obtener conocimiento. La verdad científica no consiste en afirmaciones correspondientes a datos reales del entorno, sino que es un medio de comunicación simbólicamente generalizado. El código al que la verdad se refiere para producir comunicaciones es la distinción entre verdadero y no verdadero: estos dos valores califican como científica cualquier comunicación observable con base en ellos. Lo cual significa que ha de ser tenido como científico aun el saber considerado científicamente como no verdadero" (Corsi et al., 2006, p. 48).

3 "La observación es un modo específico de operación que utiliza una distinción para indicar un lado o el otro de la distinción misma: se da una observación cada vez que un sistema opera con base en distinciones, y puede obtener y transformar informaciones. Se trata de la operación característica de los sistemas de sentido, que les permite remitir a otras posibilidades más allá del dato de vez en cuando actualizado. [...] La observación puede identificar los objetos, y (si se orienta por ejemplo a una distinción sistema/entorno) puede distinguir los procesos internos de un sistema de aquello que no le pertenece, puede identificar relaciones causales entre interior y exterior, puede atribuirles finalidad" (Corsi et al., 2006, pp. 160-161).
} 
una constelación conceptual, donde podrá al fin tomar forma compleja”, es decir, en referencia a "la problemática del todo (el todo no es todo), la problemática de la organización, el dasein físico del sistema (su situación en un entorno y en el tiempo), y la relación del sistema con el observador/conceptuador" (Morin, 1977, p. 151). De esta forma, se supera la relación epistemológica clásica sujeto/objeto -donde la causalidad es lineal y unidireccionalpor una de carácter sistémico-compleja, donde un cambio en la parte afecta al todo, la causalidad es reticular, y la construcción del conocimiento científico es un proceso determinado sociohistórica y culturalmente, como se representa en la Figura 1.

Figura 1

Nociones básicas de la relación de conocimiento en la epistemología clásica y la epistemología sistémico-compleja

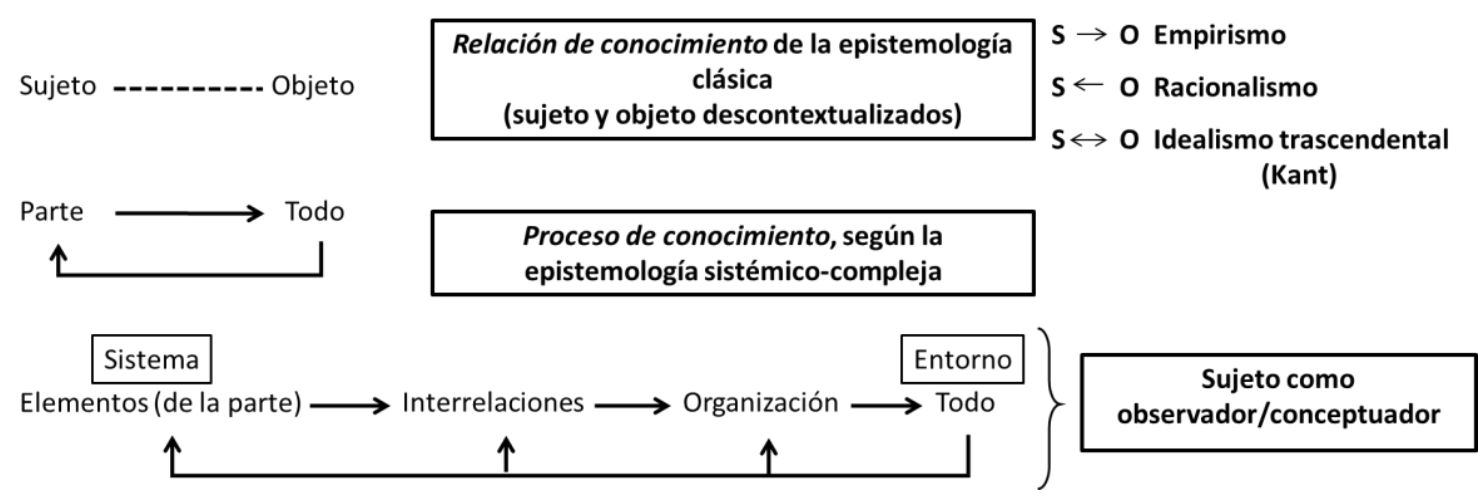

Fuente: Adaptado de Morin (1977, pp. 150-151) y Salas (2013).

En la epistemología sistémico-compleja, para el caso de los sistemas autorreferenciales ${ }^{4}$ y autopoiéticos ${ }^{5}$, las situaciones-problema bajo estudio no se describen y explican reduciéndolas a su mínima expresión, ni simplificando su estructura y

\footnotetext{
${ }^{4}$ Por sistemas autorreferenciales se entienden aquellos que "tienen la capacidad de entablar relaciones consigo mismos y de diferenciar esas relaciones frente a las de su entorno. Esta tesis abarca el sistema como un hecho y a las condiciones de su descripción y análisis mediante otros sistemas (a su vez autorreferenciales)" (Luhmann, 1998, p. 38). "[El entorno (Umwelt)] es el conjunto de elementos que tiene influjo sobre los elementos del sistema o que son influidos por él, pero que no forman nunca parte del mismo sistema. El entorno sólo consigue su unidad a partir de la relación con el sistema, pero él mismo no es un sistema y cada sistema posee uno diferente. [...] Los sistemas no se identifican con el entorno, pero no hay sistemas sin entorno" (Luhmann, 1996, p. 18).

5 "Luhmann se inspira fundamentalmente en las obras de los biólogos chilenos Humberto Maturana y Francisco Varela. La autopoiesis es la actividad propia del sistema, es la operación mediante la que el sistema crea sus propias unidades, sus propios elementos y su propia estructura" [...] La autopoiesis es una gnoseología, una teoría del conocimiento radicalmente constructivista, que combina las aportaciones de la biología y la cibernética" (Luhmann, 1996, pp. 19-20)
} 
funcionamiento en términos de causa-efecto, sino describiendo, explicando y comprendiendo su organización y relaciones internas (estructura y funciones), y con el entorno, lo que supone reconocer la diferencia fundamental sistema/entorno. Las situaciones-problema y los fenómenos bajo estudio solo se pueden describir, explicar y comprender por medio del esclarecimiento de los orígenes y efectos verosímiles que subyacen a su organización y funciones internas, y a sus relaciones con el entorno; por ende, sus resultados 0 consecuencias son multicausales y están anclados en un contexto determinado, en el cual son posibles.

Desde esa perspectiva, es válido considerar que las habitaciones de una casa son parte de ella, pero también lo son las piedras del jardín y el polvo que se acumula en el piso, los rincones y los muebles (Luhmann, 1998). El "sistema ciencia" está estructural y funcionalmente constituido para desarrollar niveles de abstracción y análisis teóricoconceptual sobre otros sistemas, "bajo aspectos que para ellos mismos no son accesibles" (Luhmann, 1998, p 38), pero esta capacidad depende del grado de avance del conocimiento y desarrollo científico de la teoría de que se trate, que cambia con el paso del tiempo. El sistema ciencia evoluciona, de la misma forma en la que evolucionan todos los sistemas autorreferenciales. En términos lógico-conceptuales, la teoría de sistemas:

Por su trasfondo epistemológico [...] es más una propuesta para el diseño de metodologías de investigación que una teoría de la lógica relacional de los sistemas, que rompe con la perspectiva epistemológica de la ciencia moderna que privilegia el método científico y su correspondiente metodología de investigación, como la única forma posible de producir conocimiento científico. Al contrario de lo que ocurre en la epistemología de las ciencias empírico-analíticas, la teoría de sistemas no formula ni prueba hipótesis, ni busca únicamente la explicación causal o cuasi explicativa, sino que descubre, describe y explica relaciones. Ello requiere, además de la descripción y la explicación (Erklären), la interpretación y la comprensión (Verstehen) de los fenómenos (Mardones, 1991), entendidos como sistemas que se relacionan consigo mismos y el entorno. (Salas, 2013, p. 70)

En el enfoque sistémico-complejo no es posible producir conocimiento científico a partir de la premisa de la reducción y la simplificación de la epistemología empírico-analítica, sino de la complejidad y la reducción de complejidad: 
Los sistemas existen gracias a la complejidad organizada, entendida como complejidad de relaciones selectivas entre los sistemas. Los sistemas no se relacionan con el entorno de manera indeterminada, sino que llevan a cabo aquellas interacciones que les permiten mantener la diferencia. La complejidad se puede reducir únicamente cuando el "complejo de relaciones de un entramado complejo es reconstruido mediante un segundo entramado de relaciones menores. Solo la complejidad puede reducir complejidad" (Luhmannn, 1998, p. 49 citado en Salas, 2013, p. 74)

De acuerdo con una concepción de la realidad, en términos de complejidad creciente, la hipercomplejidad se caracteriza por ser dialógica, recursiva y hologramática (Luhmann, 1998; Morin, 1977, 2000). El carácter dialógico hace referencia a "la dualidad en el seno de la unidad. Asocia dos términos a la vez complementarios y antagonistas" (Morin, 2000, p. 106). Por su parte, "un proceso recursivo es aquél en el cual los productos y los efectos son, al mismo tiempo, causas y productores de aquello que los produce" (ídem). El carácter hologramático alude al hecho de que "en un holograma físico, el menor punto de la imagen del holograma contiene la casi totalidad de la información del objeto representado. No solamente la parte está en el todo, sino que el todo está en la parte" (Morin, 2000, p. 107). Por ello, se afirma que una visión simple y simplificada distorsiona la realidad, ya que "lo simple no es más que un momento arbitrario de abstracción arrancado a las complejidades, un instrumento eficaz de manipulación que lamina una complejidad" (Morin, 1977, p. 425). La realidad es compleja y, en consecuencia, no se puede simplificar.

\section{Niveles de análisis de la formación profesional docente a partir del enfoque sistémico-complejo de Luhmann}

Según Luhmann (1998), para construir un modelo general de niveles de análisis de la realidad humana. El primer nivel estaría conformado por los sistemas en general. El segundo nivel, muestra la diferenciación estructural-funcional entre los sistemas básicos de la existencia de nuestra especie y su entorno, para acceder al plano elemental de la "condición humana", que se compone de máquinas, organismos, sistemas sociales y sistemas psíquicos. Las máquinas son resultado de la producción de medios y artefactos derivados de la actividad humana, y constituyen el sistema de reproducción de la vida material. 
Los organismos son los seres vivos que conforman el entorno natural del ser humano, y constituyen el sistema de reproducción de la vida natural. Los sistemas sociales forman el plexo de posibilidades de la comunicación humana y configuran el sistema de reproducción de la vida social. Los sistemas psíquicos hacen viable al ser humano individual, a partir del desarrollo de la actividad consciente, que conforma el sistema de reproducción de la conciencia. El tercer nivel de análisis representa la diferenciación de los sistemas sociales, en términos de interacciones, organizaciones y sociedades de diversa índole, como se representa en la Figura 2.

\section{Figura 2}

Niveles de análisis de la teoría general de sistemas

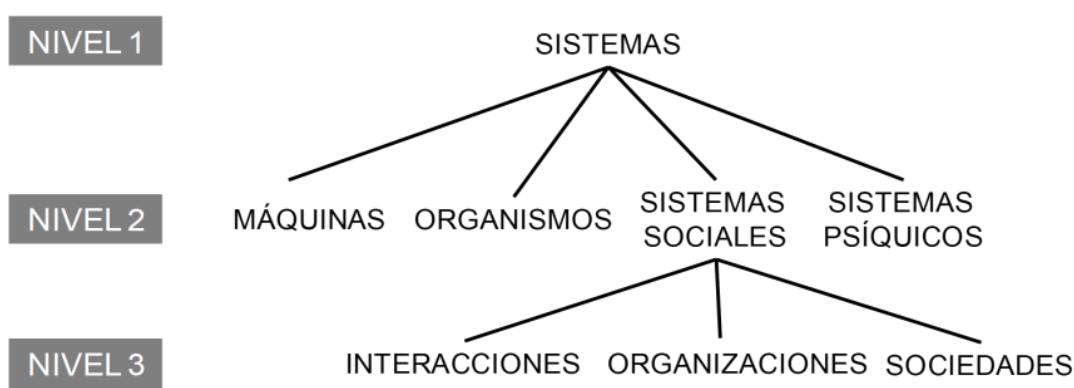

Fuente: Luhmann (1998, p. 27).

En el plano teórico-conceptual, las distinciones por niveles entre los sistemas determinan "las posibilidades de comparación [entre ellos] de una manera fecunda" (Luhmann, 1998, p. 29), con la posibilidad de que las igualdades detectadas sean llevadas al nivel de análisis superior, y las parciales solo sean válidas para el nivel de comparación de que se trate, con el valor agregado de que ello permite la distinción entre cada sistema y su entorno y, en consecuencia, la diferenciación de las posibilidades de organización y reorganización del sistema para responder a los cambios internos y del entorno.

La diferenciación descrita de primer y segundo orden hace posible el avance teóricoconceptual para la mejor comprensión de los sistemas, a partir de las distinciones entre las partes, elementos y relaciones bajo estudio. De esta forma, se asume que la teoría general de sistemas es una superteoría con pretensiones universalistas, es decir, un marco de referencia teórico-conceptual y metodológico aplicable a cualquier campo del conocimiento, que rompe con la distinción clásica entre las ciencias naturales, formales y sociales; y, más relevante todavía, que "incluye tanto a sus adversarios como a sí misma" (Luhmann, 1998, p. 29). Es decir, el marco de referencia teórico-conceptual y metodológico de avances 
epistemológicos y teóricos previos puede ser resignificado y enriquecido en sus posibilidades de procesamiento de la información y la reducción de complejidad en la Teoría General de los Sistemas Sociales.

Según la lógica relacional de los sistemas sociales, es posible construir un cuarto nivel de análisis para representar la diferencia entre organizaciones claves para la existencia humana, según sea su objetivo: productivo, educativo o político cultural, para distinguirlas con precisión entre ellas y respecto de su entorno. Para efectos de la situación problema bajo estudio, en nuestro caso, la formación profesional docente, un modelo de niveles de análisis subsecuentes permite la construcción de un quinto nivel, usando como referencia las organizaciones educativas, que incluyen el sistema de trabajo docente, el sistema educativo y el sistema de enseñanza y aprendizaje. En un sexto nivel de análisis, empleando como referencia el sistema de trabajo docente, se diferencian el sistema de formación docente, el sistema de la comunidad profesional docente y el sistema de la práctica docente (actividad docente), como se muestra en la Figura 3.

Figura 3

Niveles de análisis del sistema de trabajo docente, con base en Luhmann (1998)

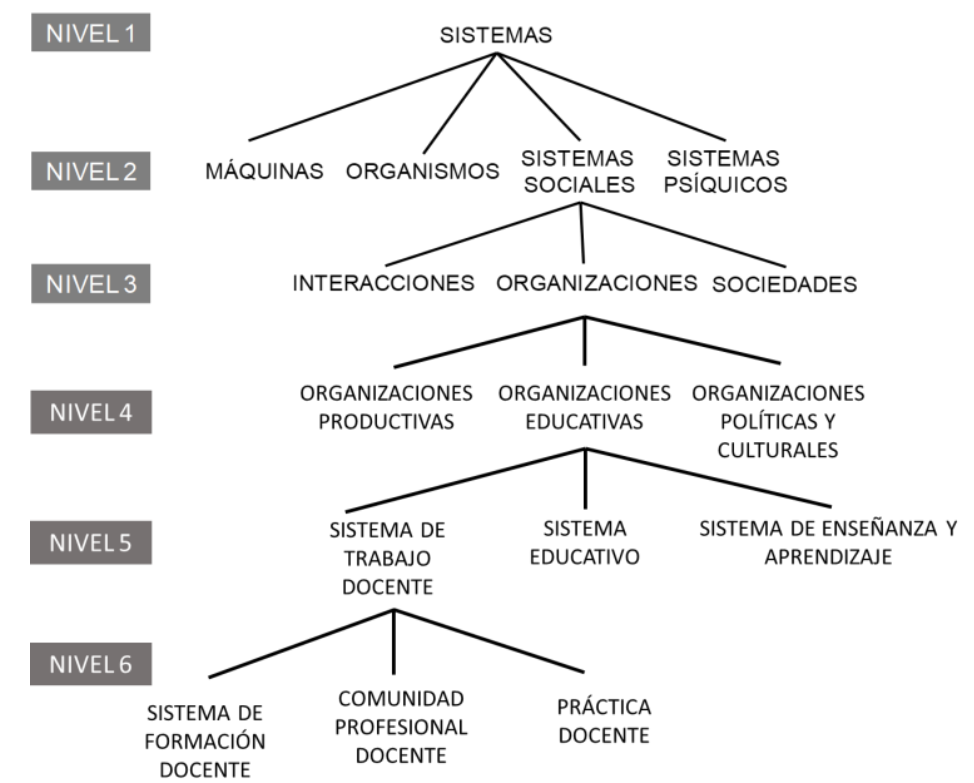

Fuente: Elaboración propia con base en Luhmann (1998, p. 27). 
En el sexto nivel de análisis aparece, como eje articulador primordial, el sistema de la comunidad profesional docente (comunidad científica de la Educación), y como interrelaciones claves entre los sistemas de este nivel, el sistema de formación docente y el sistema de la práctica docente, entendida como "ejercicio de la profesión". Finalmente, al construir un séptimo nivel de análisis, se delimita el sistema de la formación profesional docente, con su propia secuenciación en los niveles de grado, posgrado y en servicio, con la formación de grado como eje articulador primordial. Como interrelaciones claves entre los sistemas de este nivel, se observan las del sistema de formación de posgrado y el sistema de formación en servicio. Cada nivel de formación tiene especificidad y autonomía relativa respecto de los otros, así como relaciones específicas con el entorno.

La formación profesional docente de grado es el eje articulador primordial en el nivel de análisis siete (Figura 4), ya que en ella se ofrece la enseñanza científica básica en el campo, que constituye el núcleo teórico-conceptual de la Educación, donde se habilita a la persona estudiante en los fundamentos teóricos, conceptuales y prácticos que conforman el conjunto de conocimientos, métodos de investigación y aplicaciones, producto del avance científico de la disciplina y de otros campos del conocimiento que nutren su especificidad, cuya convergencia da como resultado el perfil profesional en educación.

En general, el currículo en el nivel de análisis 7 incluye sólidas bases en Filosofía -especialmente en Epistemología y Ética-, Teoría de la Educación y del currículo, así como conocimientos de orden superior en Investigación educativa, Historia del pensamiento en Educación -nacional y mundial-, Psicología Educativa -haciendo énfasis en el grupo de edad que corresponda-, Sociología y Antropología de la Educación, Economía de la Educación, Política Educativa -nacional y mundial-, Administración de la Educación y Derecho Educativo, Didáctica general y específica, y evaluación de los aprendizajes, entre los más importantes. 
Figura 4

Niveles de análisis del sistema de formación docente, con base en Luhmann (1998)

NIVEL 6

NIVEL 7

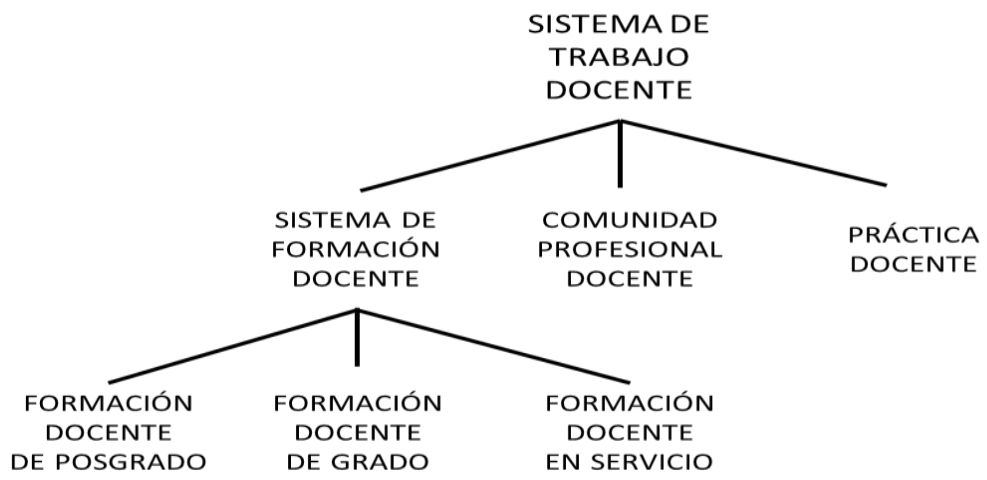

Fuente: Elaboración propia con base en Luhmann (1998, p. 27)

En la formación docente de grado, se incluye el desarrollo de un perfil académico ${ }^{6}$, entendido como el bagaje cultural necesario para una adecuada integración de los conocimientos disciplinares y profesionales en un contexto sociocultural y laboral.

6 De acuerdo con Glazman (2001, pp. 132-133), "El desarrollo y ejercicio de las capacidades cognoscitivas proporcionan al egresado los medios para solucionar problemas. Las habilidades académicas favorecen aprendizajes y capacitan para el dominio de la comunicación oral y escrita de los aspectos técnicos y conceptuales de su profesión. Las actitudes y las habilidades de interacción están relacionadas con la capacidad de trabajo en grupo, la actitud cooperativa y el ejercicio ético de la profesión. Las categorías que se incluyen en el perfil académico y sus principales componentes son:

1. Desarrollo intelectual: razonamiento, pensamiento crítico, desarrollo y estímulo de la creatividad, concepciones filosóficas, métodos y técnicas de investigación, habilidad para resolver problemas.

2. Capacidades académicas: habilidad para la comunicación en la lengua básica, en forma verbal y escrita, comunicación verbal y escrita en otros idiomas, uso y conocimiento de la informática, autoaprendizaje, diseño y transmisión de elementos y productos relacionados con la profesión (material científico y técnico).

3. Desarrollo de actitudes y valores: interacción socioprofesional (trabajo en equipo, liderazgo, cooperación, desarrollo de especialidades), ética profesional, académica y social, conciencia ética de la profesión.

4. Apreciación cultural y artística, reflexión y crítica del ejercicio profesional en las condiciones de la realidad nacional y mundial, interacción con la comunidad profesional.

5. Dominio sociohistórico: valores socioculturales e históricos, análisis de las condiciones políticas nacionales, problemas de la sociedad contemporánea (ecológicos, sociales, morales, laborales), deontología de la profesión, profesión y ambiente (desarrollo sustentable).

En el enfoque por competencias, cuando se separa el perfil de salida de la educación superior entre competencias específicas y competencias genéricas, en las segundas se hace referencia al perfil académico. Se asume que "las competencias genéricas identifican los elementos compartidos, comunes a cualquier titulación, tales como la capacidad de aprender, de tomar decisiones, de diseñar proyectos, las habilidades interpersonales, etc." (Beneitone et al, 2007, p. 37) 
La formación en el "núcleo duro" de la disciplina hace posible que el estudiantado conozca y comprenda la dimensión teórico-explicativa de la Educación y el ejercicio de la profesión, la especificidad del fenómeno educativo, las situaciones que estudia y sobre las que interviene, con el propósito de confirmar sus modelos y teorías y, eventualmente, ampliarlos y reelaborarlos, lo que supone procedimientos de ajuste, producto del avance del conocimiento en el campo disciplinar. También, desarrolla el conocimiento y la comprensión de la dimensión tecnológico-instrumental de la Educación, que construye y valida procedimientos e instrumentos de aplicación. Es decir, la enseñanza, en tanto práctica concreta y diferenciable de la práctica en cualquier otro campo profesional, que responde a la diferencia ser docente/no ser docente.

La formación de grado, además, debe habilitar a la persona profesional en Educación en el conocimiento de la dimensión técnico-práctica, para la intervención directa sobre las actividades específicas a las que se aboca, que depende de la edad, características y grado de desarrollo sociocultural y cognitivo de la persona aprendiz. Por lo general, este nivel de formación requiere de cinco años de estudio -en Costa Rica equivalen a 3 años de Bachillerato y 2 años de Licenciatura-, que incluyen cursos prácticos supervisados por personal docente y profesionales en servicio a lo largo del avance en la carrera.

La naturaleza de la profesionalización en Educación requiere de la práctica supervisada, al menos en el último año de formación de Licenciatura, y la elaboración de un trabajo final de graduación, donde se aplican los conocimientos teórico-conceptuales, prácticos y en investigación. La práctica docente permite al estudiantado aproximarse a una situación-problema del campo de conocimiento del nivel educativo en el que se forma, ya sea preescolar, primaria, educación secundaria, administración educativa, educación superior o educación no formal. Como se puede observar, la formación de grado en Educación es clave, ya que en ella se logra el perfil profesional y académico idóneo para el ejercicio de la profesión docente, cuya titulación es requisito para ello.

En el nivel de posgrado, se da la formación especializada en el campo de la Educación para la obtención del título de maestría o doctorado. El primero, generalmente, tiene una duración de 2 años. En el Doctorado, con una duración de 4 a 5 años, se hace énfasis en investigación educativa e interesa el avance teórico en el campo disciplinar. En la formación docente en servicio, se busca la actualización en el núcleo teórico-conceptual de la Educación, como en áreas de especialización y subespecialización. Incluye la puesta en 
práctica de innovaciones educativas y didácticas, la adaptación del profesorado al contexto nacional e institucional y a reformas educativas, cambios en el currículo y la gestión educativa, el desarrollo de capacidades, actitudes y habilidades blandas, así como la implementación de nuevos roles del personal docente, entre los más importantes.

\section{Aristas de la formación docente: Algunos problemas que "vemos" y "reconocemos"}

El enfoque epistemológico de la formación docente que predomina en la actualidad es el empírico-analítico, ya que se le concibe como un sistema cerrado; es decir, como un sistema que "no puede dar acogida a ningún tipo de input que no esté contenido dentro de sí mismo. De allí surge, inexorable, la ley de la entropía de la termodinámica” (Luhmann, p. 18), entendida como el caso límite en el que:

... una información acerca de un elemento no permite sacar ninguna conclusión acerca de otros. El sistema es entrópico para sí mismo cuando en el proceso de la reproducción, es decir, de la sustitución de elementos que deben ser sustituidos, cualquier elemento de posible sucesión tiene la misma probabilidad. Dicho de otro modo, la entropía carece de todo estrechamiento de la capacidad de sucesión, con lo cual falta también la ganancia de tiempo que resulta del hecho de que no todo viene al caso. El término denomina entonces el caso límite en el cual la reproducción del sistema se convierte en casualidad a partir de sí mismo. (Luhmann, 1998, p. 69)

La entropía constituye un momento particular de los sistemas con complejidad temporalizada ${ }^{7}$, a partir del cual éstos pierden la capacidad de restructurarse a sí mismos, a

\footnotetext{
7 De acuerdo con Luhmann (1998), "puede presuponerse la complejidad temporalizada que, momento a momento, selecciona sus propias condiciones y puede diferenciarse por ello" (p. 204); esto es, los sistemas complejos siempre están en referencia al tiempo, entendido como "la observación de la realidad con base en la diferencia entre pasado y futuro. Cada sistema existe siempre sólo en el presente y simultáneamente con el propio entorno: en este sentido, pasado y futuro no son puntos de partida o de llegada, sino horizontes de posibilidad. Para los sistemas constitutivos de sentido, la realidad se presenta como diferencia entre actualidad y potencialidad y uno de los modos para hacer accesible al sistema la potencialidad es el tiempo, el cual se constituye redoblando la distinción: sobre el lado potencial de la diferencia entre actualidad y potencialidad se distingue ulteriormente entre pasado y futuro. [...] Para cualquier observador el tiempo está dado antes que nada por el hecho de que cualquier distinción se constituye por dos lados para pasar de uno al otro se necesita una operación y con ella se necesita también el tiempo. Con esto se genera una diferencia entre el observador mismo, que siempre está presente, y la diferencia entre antes y después ligada al evento que ha permitido pasar de un lado al otro de la distinción. La diferencia entre simultaneidad y diferencia antes/después es el tiempo". (Corsi et al., 2006, pp. 212-213)
} 
través de selecciones alternativas de elementos de su organización que deben ser sustituidos, debido a la información que tengan sobre su propio funcionamiento y el entorno. De ahí, la dependencia de los sistemas de la información sobre ellos mismos y el entorno, ya que ella "reduce la complejidad en la medida en que da a conocer una selección y, con ello, excluye posibilidades" (Luhmann, 1998, p. 84). Si en la sustitución de elementos en un sistema no es posible anticipar una probabilidad distinta respecto de los que deben ser sustituidos -porque ya no funcionan como debieran-, el sistema puede evolucionar hacia la destrucción (Luhmann, 1988), pues se vuelve impredecible para sí mismo.

El procedimiento "ensayo-error", donde de manera especulativa se seleccionan elementos alternativos, no utiliza información que reduzca complejidad, sino que el sistema evoluciona de manera entrópica, introduciendo desorden y mal funcionamiento al interior del sistema, con el respectivo deterioro de sus relaciones consigo mismo y con el entorno. La entropía evidencia falta de información para la toma de decisiones sobre las selecciones de alternativas de solución a problemas internos y de enlace o acoplamiento con el entorno.

En el enfoque empírico-analítico de la formación docente subyacen la simplificación y la desconexión de los componentes de la sociedad, el sistema educativo y el sistema de trabajo docente y, en consecuencia, entre la formación de grado, posgrado y en servicio. Cada sistema de formación profesional docente está aislado del otro, y regula sus relaciones internas y con el entorno con base en supuestos que no siempre se corresponden con la realidad propia y la de la sociedad. Esto conlleva un incremento sostenido de la complejidad interna que no encuentra salida por medio del uso de la información disponible, lo que lleva a una crisis de difícil manejo en la práctica; incluso, a crisis en el manejo de la crisis (Offe, 1990). La toma de decisiones con base en datos e información básica es fundamental para evitar el deterioro estructural-organizativo del sistema y su funcionalidad.

En la Figura 5 se representan los componentes aislados del ciclo de la formación profesional docente, vistos como entidades vinculadas entre sí de manera estereotipada, donde se observa que el sistema educativo formal está fragmentado, porque es concebido a partir de componentes aislados, que se articulan de manera mecánica y repetitiva, a partir de la obtención de certificaciones que acreditan a la persona a pasar de un nivel de formación a otro. Estos componentes están jerárquicamente organizados y tienen autonomía estructural y funcional. La formación profesional docente es postsecundaria y se lleva a cabo en instituciones de educación superior o parauniversitarias. En el nivel universitario se ofertan 
dos niveles de formación: grado y posgrado. Recientemente, las universidades incluyen programas de formación continuada que buscan la actualización profesional de la población graduada, por lo que acceden a ella profesionales en servicio.

En el sistema laboral público y privado, el sector empleador del personal docente establece los requisitos para el ejercicio de las profesiones relacionadas con la Educación y, por ello, gestiona sus propios procesos y procedimientos de selección y reclutamiento. También, proporciona la actualización profesional y la formación en servicio, debido a reformas en el sistema educativo y a la eventual incorporación de innovaciones en el proceso de enseñanza y aprendizaje, que pueden ser curriculares, didácticas, de evaluación de los aprendizajes, de la organización del trabajo docente y de gestión educativa, entre otros.

Figura 5

Componentes del ciclo de la formación docente desde el enfoque empírico-analítico

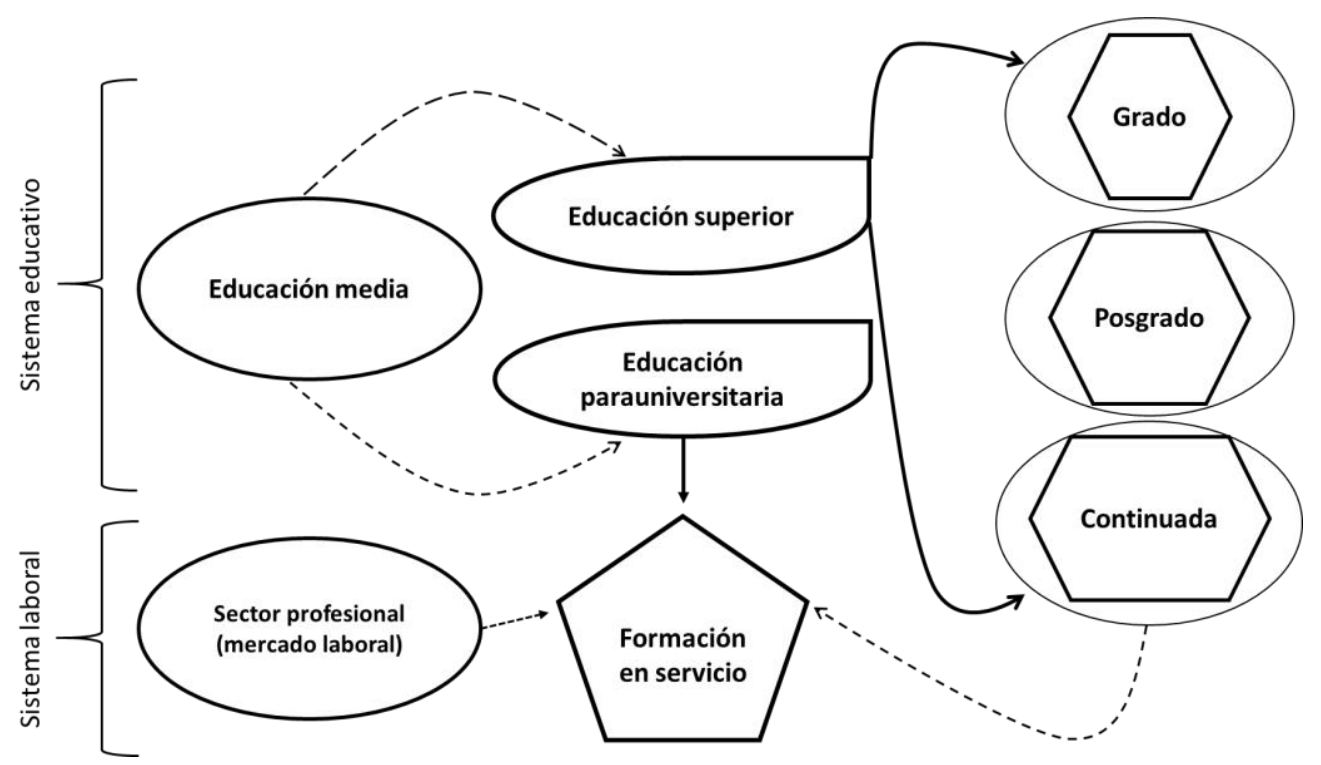

Fuente: elaboración propia, 2018.

El problema fundamental del enfoque empírico-analítico de la formación profesional en Educación es que cuando aparecen desajustes en sus componentes, no existe la capacidad en cada nivel para ahondar en sus orígenes y, en consecuencia, no se cuenta con la información necesaria, tanto interna como del contexto, para diferenciar las situacionesproblema internas de las del entorno, ni de sus enlaces -acoplamientos-, lo que impide 
introducir complejidad para reducir complejidad. Producir y utilizar información interna y del contexto para responder a los cambios del entorno es importante, ya que, "según una antigua y sabia regla, las verdades sólo aparecen en contexto, mientras que los errores aparecen en forma aislada" (Luhmann, 1998, p. 76).

Para obtener información relevante interna y del contexto es necesario un proceso constante de auto-observación y auto-descripción que permita "comprender al sistema como diferencia frente a un entorno" (Luhmann, 1998, p. 167), ya que esa diferencia conlleva las "informaciones y los puntos direccionales para la conducta de enlace" (ídem) con este. Así, por ejemplo, si se toma como eje articulador la formación docente de grado, se requiere de un currículo que prepare a la persona en los conocimientos, habilidades, capacidades y actitudes necesarias para "ser docente", entendido como "perfil de salida" de la profesión; pero, esto requiere a su vez de la delimitación de un "perfil de ingreso", lo que nos pone ante la diferencia perfil de ingreso/perfil de salida en la formación profesional docente de grado.

Es frecuente que cuando el perfil profesional de salida en el campo de la Educación no responde a las necesidades del contexto, se espera que ello se resuelva ajustándolo a la expectativa social identificada, bajo el supuesto de que ello es suficiente para solventar los vacíos identificados. Ante esto, la respuesta suele ser de naturaleza didáctico-curricular, bajo el supuesto de que la formación profesional de grado en Educación enlaza con el sistema de formación previo: la educación media, obliga a definir un perfil de ingreso acorde con el perfil de salida profesional previsto. Sin esta práctica, es probable que los desajustes entre el perfil de salida profesional y las necesidades del contexto persistan. De igual forma, se obvia que el perfil de salida profesional en Educación enlaza con el sistema de trabajo docente y el sistema educativo, que enlazan, a su vez, con la sociedad. De ahí la importancia de investigar el sistema de trabajo docente y las necesidades del contexto en materia de formación profesional docente de grado.

Un error común en el enfoque del ciclo de formación profesional en Educación es que se hacen reformas educativas para atender necesidades sociales y del mercado laboral, sin identificar con precisión el enlace que ello tiene con el sistema de trabajo docente, el enfoque curricular y el paradigma educativo, de acuerdo con la diferencia entre esos sistemas y el entorno, que en este caso supone niveles distintos de análisis. Esto es importante, ya que la "educación escolar depende primariamente de que los alumnos entiendan a los profesores" (Luhmann, 1996, p. 123). Ahora bien, si esto no ocurre significa que hay disfunciones en el 
proceso educativo, que provocan que el estudiantado no entienda lo que el profesorado le enseña. Por esto, se modifica la formación profesional docente, a fin de hacer correcciones. No obstante, se omite, al tomar esta decisión, el enlace entre la sociedad y el sistema educativo, donde, por ejemplo, los cambios en la estructura y las funciones de la familia, y los cambios en la estructura y las relaciones económicas impactan los intereses y necesidades educativas del estudiantado, lo que se refleja en la diferencia formación recibida/formación requerida. Desde esta perspectiva:

La educación se vuelve necesaria para la sociedad cuando la socialización no es suficiente para asegurar la capacidad del comportamiento adecuado. Paralelamente a la socialización, que se lleva a cabo por el simple hecho de participar de la comunicación, se desarrolla una socialización de tipo particular, intencionada y, por lo tanto, educativa: la educación nace propiamente cuando a partir de la intención pedagógica, se valora un comportamiento como adecuado. La socialización y la educación no coinciden, aunque naturalmente la socialización es presupuesto de la educación, ya que sólo aquel que está socializado puede ser educado. (Corsi et al., 2006, p. 96)

Un problema "visible" de la educación en nuestro tiempo es que existe una tendencia a la sobrecarga en el currículo, que se traduce en exceso de contenidos, ya que en los últimos treinta años el sistema educativo ha tenido que asumir una función adicional a la educativa: la terapéutica. Un ejemplo de esto son las crecientes demandas sociales por incluir en el currículo oficial temáticas extracurriculares, de naturaleza socializadora, que orientan la conducta de la población estudiantil en diferentes áreas, como, por ejemplo, vial, sexual, cívica, financiera, nutricional y consumo de drogas, lo que va en detrimento de la formación académica. El currículo escolar es cada vez más de naturaleza "extra-curricular" que académica, lo que convierte al sistema de interacción-enseñanza en un subrogado del sistema de interacción-familia y del sistema interacción-comunidad local, regional y mundial. El deterioro de la educación como experiencia personal y social de naturaleza académica, nos regresa a una pregunta de fondo que sigue sin una respuesta clara en nuestro tiempo: “¿en qué se reconoce la educación?” (Luhmann, 1996, p. 139).

La "dinámica de endoso de funciones de socialización a la educación" vuelve el proceso educativo algo adscrito a contingencias socioculturales, económicas y políticas, que 
responden a cambios en la estructura social, ante los cuales el sistema educativo se ve restringido para actuar, porque obedecen a acciones que se deben emprender en otros sistemas de socialización. Es relativamente fácil y frecuente que se transfieran al sistema educativo responsabilidades y deberes de los sistemas de socialización familiar, política y económica, bajo el supuesto de que ello solventará situaciones no deseables en la convivencia, el civismo, el desarrollo económico, la distribución de la riqueza, la productividad y la exclusión social.

Las diferencias sistema familia/sistema educativo, sistema de salud/sistema educativo, sistema de derecho/sistema educativo, sistema económico/sistema educativo se borran en el traslape de funciones entre los sistemas de socialización y el sistema educativo, con el agravante de que los problemas de índole social, económica y de salud no se resuelven por esa vía de manera efectiva, mientras se deteriora académicamente el proceso educativo, al convertirlo en actividad terapéutica y de contención psico-afectiva a escala individual y social. En este esfuerzo, por necesario que parezca, no está clara la diferencia entre la formación académica y el fortalecimiento de formas de conocimiento y conciencia de otras esferas de la vida personal y social. Si bien, la formación académica y el desarrollo cognitivo influyen en la toma de decisiones personales, no pueden agotar su potencialidad compensando interacciones desestabilizadas en otros sistemas.

\section{Puntos ciegos en el enfoque empírico-analítico de la formación docente: Algunos ejemplos}

En el enfoque sistémico-complejo existe un principio denominado "punto ciego", propuesto por Heinz von Foerster, que usa como metáfora de nuestras posibilidades de observar la realidad, lo que ocurre con la visión ocular, ya que:

Existe una zona en la retina al que corresponde, sin que el vidente se dé cuenta, un punto de ceguera. De hecho, existe un punto en la retina en el que no hay células receptoras y por esto nuestro campo de visibilidad es incompleto: no somos capaces de ver lo que recae en dicha zona y menos aún, al no conocer esta carencia, de ver que no vemos. Este principio ha sido abstraído y aplicado a observaciones de todo tipo, las cuales, orientándose a una distinción específica, no son nunca capaces de observar la distinción misma. Si se orienta la observación a la distinción verdadero/no verdadero, por ejemplo, no es posible observar si tal distinción es a su vez verdadera o 
no verdadera: representa el punto ciego de la observación en cuestión. (Corsi et al., 2006, p. 69)

En el enfoque empírico-analítico de la formación docente, encontramos "puntos ciegos" en los niveles de grado, posgrado y educación continuada, que es indispensable reconocer y atender desde un enfoque sistémico-complejo, a fin de explorarlos en el ámbito de la investigación y la práctica, como un fenómeno complejo que nos permita desarrollar la capacidad de producir y utilizar la información necesaria para tomar decisiones y reducir complejidad en sus procesos, enlaces y relaciones.

Respecto de los ejemplos de enlace entre los niveles de formación profesional docente señalados anteriormente, es clave la definición de un perfil de ingreso para las personas candidatas a formarse en Educación, debido a que se requiere de un sistema de selección adecuado. Esto, a su vez, conlleva la vinculación entre el sistema de educación superior y el sistema de educación media, que permita procesos de selección efectivos, tanto en el nivel vocacional como en relación con la formación general básica. Al respecto, las pruebas de admisión a las universidades evalúan conocimientos y habilidades cognitivas generales, que son predictores del éxito académico en educación superior, pero no predicen la vocación ni ayudan a identificar los intereses de las personas por estudiar una determinada carrera. Esto es un indicador de la necesidad de hacer pruebas especiales para ingresar a las carreras de educación, a fin de identificar los intereses vocacionales de las personas interesadas. Esto no es trivial, ya que determina el éxito y rendimiento académico en la universidad, así como el nivel de logro a alcanzar por la persona en su futuro profesional y sociolaboral.

En Costa Rica, un desafío para las universidades que cuentan con este tipo de prueba es elevar la nota de admisión para las carreras de Educación e implementar pruebas especiales, que podrían consistir en la elaboración de ensayos sobre los intereses vocacionales en el campo, donde la persona exponga sus motivaciones para emprender sus estudios, así como sus expectativas profesionales y laborales en el campo de la Educación. Las personas que resulten elegibles, de acuerdo con los criterios establecidos de forma y fondo de la prueba especial, se podrían someter, adicionalmente, a una entrevista donde personal académico especializado en Educación comente con cada estudiante su ensayo, para profundizar en sus motivaciones, intereses y expectativas socio-profesionales y laborales. 
La metodología de selección descrita introduce complejidad al proceso de selección de personas para ingresar a las carreras de Educación, pero reduce complejidad en la formación profesional porque permite elegir población estudiantil con vocación e interés por la profesión, que, además, cuenta con el nivel de conocimientos generales suficientes para tener éxito en sus estudios superiores y especializados. La diferencia entre perfil de ingreso a carrera/perfil de salida profesional es fundamental en la formación de grado, así como para otros niveles de formación.

En relación con el perfil profesional de salida, se deben considerar las necesidades del contexto, así como el proceso de actualización y revisión permanente de los contenidos curriculares del núcleo duro de la disciplina. Para esto, la investigación científica sobre el desarrollo teórico y hallazgos de investigación pura y aplicada en el campo, y los estudios de seguimiento de población graduada son herramientas fundamentales para obtener información clave, orientada a la reducción sistemática de complejidad en el enlace del plan de estudios con el avance de la disciplina y el perfil profesional de salida, con las necesidades del contexto; en particular, del sistema educativo y del sistema de trabajo docente.

El desarrollo de la comunidad de profesionales en educación es fundamental en la revisión y actualización de los planes de estudio, de ahí la importancia de los congresos, seminarios, conferencias y conversatorios con personas expertas, entre otras estrategias de vinculación de las universidades con la comunidad profesional y la población graduada.

El enlace entre los sistemas de formación profesional en Educación supone la diferencia perfil de ingreso a carrera/perfil profesional de salida, currículo oficial/avances en la disciplina, perfil de salida profesional/necesidades del contexto. La necesidad de definir perfiles de ingreso y salida, como se mencionó, atraviesa todos los sistemas de formación profesional. De igual forma, el avance de la disciplina impacta al currículo oficial y, por ende, el perfil de salida que, a su vez, debe responder a las necesidades del contexto, como se muestra en la Figura 6, donde la vinculación mediante el perfil de ingreso a carrera/perfil profesional de salida operan como enlaces entre los sistemas, que producen información para reducir complejidad. 
Figura 6

El perfil de ingreso y de salida profesional como enlace y corrección del enfoque sistémico-complejo de la formación docente

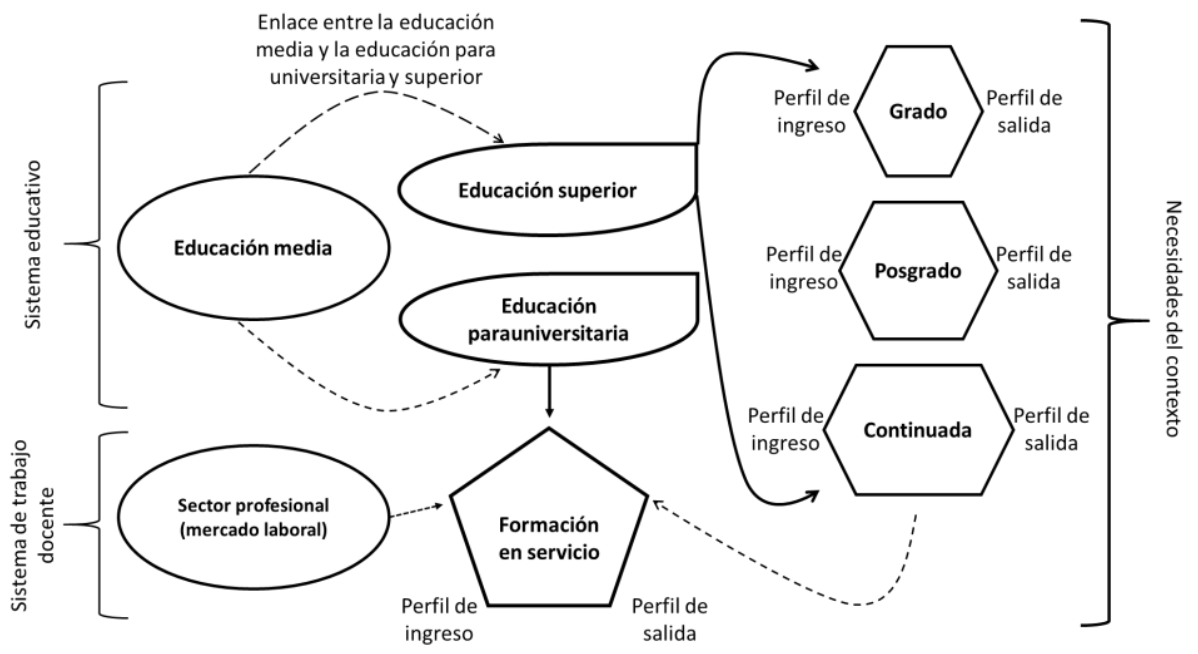

Fuente: elaboración propia, 2018.

La información para la reducción de complejidad funciona en cada sistema y en el entorno de la formación profesional docente; es decir, mejora los enlaces entre los niveles de formación, con el respectivo beneficio en términos estructural-funcionales internos y externos. Esto garantiza una articulación selectiva entre los niveles de la formación docente, donde se incrementan las posibilidades de coherencia a partir de las diferencias propias entre perfil de ingreso a carrera/perfil de salida profesional, currículo oficial/avances en la disciplina, perfil de salida profesional/necesidades del contexto. La incorporación de mecanismos de índole sistémico-compleja en el proceso de la formación profesional docente tiene un impacto en cadena al interior de cada sistema de formación y en el entorno, porque permite que cada sistema se acople estructuralmente al contexto; es decir, favorece la capacidad adaptativa de cada sistema de formación profesional docente a su entorno.

\section{Conclusiones}

El cambio de enfoque empírico-analítico al enfoque sistémico-complejo de la formación profesional docente tiene profundas implicaciones teórico-conceptuales, prácticas, organizativas, culturales y funcionales, pues afecta de manera directa a la comunidad profesional, al sistema educativo y al sistema de trabajo docente, en la media en que le da 
forma compleja y la saca de una perspectiva simple y simplificada. Nuestra tesis es que esta tarea es posible y empieza introduciendo correcciones que favorezcan el enlace entre los sistemas y niveles de la formación profesional docente, de los cuales se mencionaron algunos, que son parte del problema de aislamiento y desarticulación entre los sistemas de formación docente vigentes.

En la actualidad, en el mundo se ensayan cambios e innovaciones en la formación docente, que tienen un impacto limitado en el sistema educativo y la trasformación en la enseñanza y el aprendizaje, ya que responden a medidas curriculares aisladas y descontextualizadas. El problema, entonces, no es identificar "áreas de mejora", sino encontrar mecanismos que brinden la información suficiente para orientar la toma de decisiones y hacer selecciones de enlace pertinentes entre los niveles de formación profesional docente, como en el caso del control del perfil de ingreso a las carreras de Educación, los cambios curriculares y los perfiles profesionales de salida, en atención a las dinámicas propias de los sistemas de formación, el avance en la disciplina y las necesidades del contexto.

En el enfoque sistémico-complejo de la formación docente, se reconoce que la única forma de reducir complejidad es introduciendo complejidad, es decir, desarrollando el conocimiento y la información suficiente sobre las especificidades de cada nivel de formación y de los sistemas de influencia inmediata: el núcleo teórico-conceptual y el avance de la disciplina, el desarrollo del currículo para la formación profesional en Educación -que incluye el perfil de ingreso a carrera y el perfil profesional de salida-, y las necesidades del contexto. De esta forma es factible ajustar en un tiempo razonable, la permanente tensión entre los sistemas y niveles de formación y el entorno, resultado de la diferencia sistema/entorno. Esto se debe a que la investigación en Educación y el aprovechamiento de sus hallazgos en la disciplina y otros campos del conocimiento afines son componentes claves en la reducción de complejidad. No obstante, en este ámbito es donde hay mayores debilidades, porque se hace énfasis en el ajuste del currículo al contexto, pero se omiten los componentes teóricoconceptuales, el sector laboral y el avance de la disciplina. Además, es frecuente que la formación de grado sea débil en el desarrollo de conocimientos, habilidades y actitudes para la investigación.

Una universidad que no invierte en investigación sobre ella misma y sus procesos, no es competente para responder a la complejidad interna y del entorno, ya que con ello 
compromete su pertinencia y calidad. La capacidad de enlace de los sistemas favorece la auto-organización y la autopoiesis y, con ello, la posibilidad de reproducción del sistema, es decir, su existencia y funcionalidad. Desde esta perspectiva, los mecanismos de enlace entre los distintos niveles de la formación profesional docente favorecen el encadenamiento entre ellos y, por ende, su pertinencia y calidad. Esto es de gran importancia, máxime si se considera la relevancia que ha adquirido el aseguramiento de la calidad de la educación superior por medio de procesos de autoevaluación y acreditación interna y con organismos externos, tanto nacionales como internacionales.

En la actualidad, el enfoque empírico-analítico de la formación docente ha demostrado ser insuficiente para atender la complejidad creciente que caracteriza a las organizaciones en la sociedad contemporánea. Alvin Toffler (1995) señaló que:

Como norma, las grandes organizaciones introducen cambios significativos sólo cuando se dan ciertas condiciones previas. Primero, debe haber enormes presiones externas. Segundo, debe existir gente dentro que esté muy descontenta del orden existente. $Y$ tercero, debe haber una alternativa coherente incorporada al plan, modelo o visión. (pp. 22-23)

Es evidente que esas condiciones están dadas en el ámbito de la formación docente y que el enfoque sistémico-complejo es un potente instrumental epistemológico, teórico, conceptual y práctico, que se puede incorporar con relativa facilidad, como otra visión y un nuevo modelo para atender la diversidad y complejidad de las demandas educativas emergentes, para dar respuesta a la enorme presión externa sobre el cambio en Educación. Asimismo, el descontento interno en las organizaciones educativas obedece, en gran medida, a la imposibilidad de encontrar soluciones simples, efectivas y de rápida implementación en el sistema educativo en general y en la formación docente en particular. Es difícil no malograr el "vino nuevo en odres viejos", pero es posible hacer cambios estratégicos con gran impacto en la mejora estructural y funcional de los sistemas de formación profesional docente. 


\section{Referencias}

Beck, Ulrich. (1998). ¿Qué es la globalización? Falacias del globalismo, respuestas a la globalización. España: Paidós.

Beck, Ulrich., Giddens, Anthony. y Lash, Scott. (2001). Modernización reflexiva. Política, tradición y estética en el orden social moderno. Madrid, España: Alianza Universidad.

Bell, Daniel. (1994a). El advenimiento de la sociedad post-industrial. Un intento de prognosis social. Madrid: Alianza.

Bell, Daniel. (1994b). Las contradicciones culturales del capitalismo. Madrid: Alianza.

Beneitone, Pablo., Esquetine, César., González, Julia., Maletá, Maida., Siufi, Gabriela. y Wagenaar, Robert. (2007). Tuning Amércia Latina. Reflexiones y perspectivas de la Educación Superior en América Latina 2004-2007. España: Universidad de Deusto.

Castells, Manuel. (2000a). The Rise of the Network Society. The Information Age: Economy, Society and culture. Great Bretain: Padstow \& Conrwall. Vol I.

Castells, Manuel. (2000b). End of Millennium. The Information Age: Economy, Society and culture. Great Bretain: Padstow \& Conrwall. Vol. III.

Castells, Manuel. (2001). The Internet Galaxy. Reflections on the Internet, Business, and Society. New York: Oxfort University Press.

Corsi, Giancarlo., Esposito, Elena. y Baraldi, Claudio. (2006). Glosario sobre la teoría social de Niklas Luhmann. México: Universidad Iberoamericana.

García, José Luis. (1999). Formación del profesorado. Necesidades y demandas. Barcelona: Praxis.

Glazman, Raquel. (2001). Evaluación y exclusión en la enseñanza universitaria. México: Paidós Educador.

Guidens, Anthony. (1995). Modernidad e identidad del yo. El yo y la sociedad en la época contemporánea. Barcelona: Península.

Luhmannn, Niklas. (1996). Teoría de la sociedad y pedagogía. España: Paidós.

Luhmannn, Niklas. (1997). Observaciones de la modernidad. España: Paidós.

Luhmannn, Niklas. (1998). Sistemas Sociales. Barcelona: Anthropos.

Morin, Edgar. (1977). El método. La naturaleza de la naturaleza. Madrid: Cátedra.

Morin, Edgar. (2000). Introducción al pensamiento complejo. Barcelona: Gedisa. 
Offe, Claus. (1990). Contradicciones en el Estado de Bienestar. Madrid: Alianza.

Salas, Flora. (2013). La integración de las TIC en la docencia en la UCR: un enfoque sistémico-complejo del personal docente que se certificó en el curso Educar para el Futuro, versión universitaria en el período 2003-2006 (Tesis doctoral). Universidad de Costa Rica, Ciudad Universitaria Rodrigo Facio, Costa Rica.

Toffler, Alvin. (1995). La empresa flexible. España: Plaza \& Janes. 


\section{Revista indizada en}
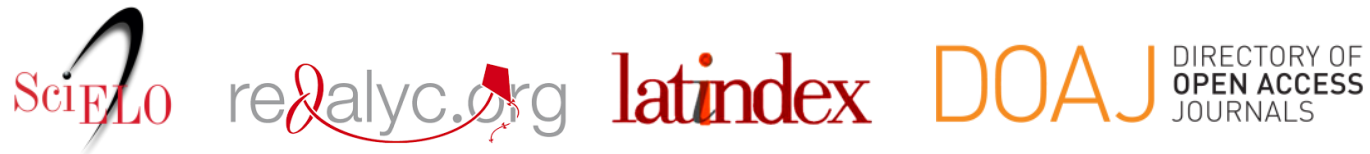

Distribuida en las bases de datos:

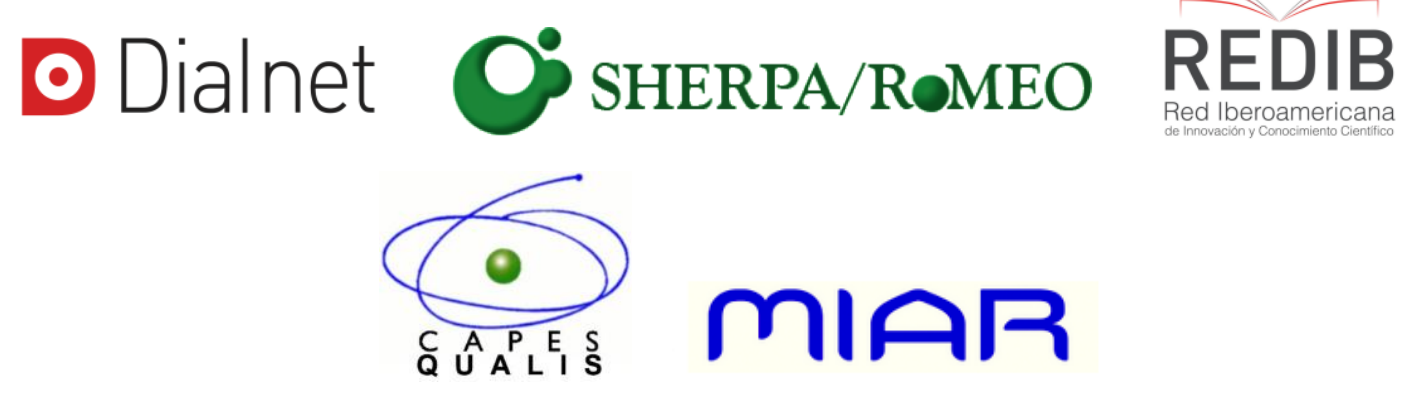

